
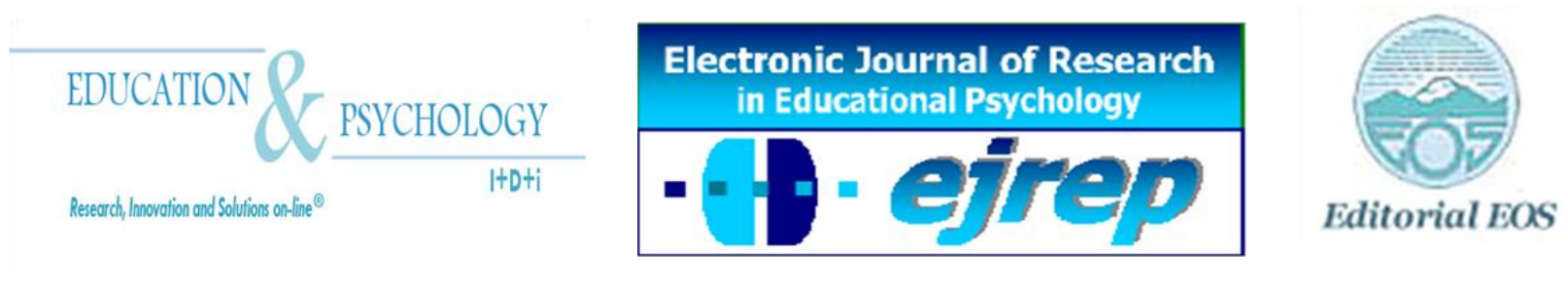

\title{
Perfil psicosocial de adolescentes con aptitudes sobresalientes de un bachillerato público
}

\section{Gabriela Ordaz Villegas ${ }^{1}$ y Guadalupe Acle Tomasini ${ }^{2}$}

\author{
${ }^{1}$ Facultad de Psicología, \\ Universidad Nacional Autónoma de México, Distrito Federal \\ ${ }^{2}$ Facultad de Estudios Superiores Zaragoza, \\ Universidad Nacional Autónoma de México, Distrito Federal
}

\section{México}

Correspondencia: Gabriela Ordaz Villegas. Juan de la Barrera 59-17, Col. Darío Martínez, Valle de Chalco, Estado de México. México. E-mail: gabordaz@yahoo.com.mx

(C) Education \& Psychology I+D+i and Editorial EOS (Spain) 


\section{Resumen}

Introducción. Los modelos actuales abocados al estudio de las aptitudes sobresalientes como el de la Interdependencia Triadica, definen a éstas como el resultado favorable de la interacción entre factores intrínsecos (habilidad intelectual, creatividad y motivación) y extrínsecos (familia, amigos y escuela). Al tomar en cuenta lo anterior el objetivo del presente trabajo consistió en identificar y establecer un perfil de adolescentes con aptitudes sobresalientes en el que se incluyeran tanto características intrínsecas como extrínsecas así como analizar las relaciones y las diferencias respecto de estas características al interior del grupo resultante en dicho perfil.

Método. La muestra estuvo constituida por 666 alumnos de una escuela pública, las edades fluctuaron de 12 a 18 años con una media de 14.77. Se realizaron diversos análisis estadísticos como correlación de Pearson, $t$ de Student y ANOVA para contrastar las posibles relaciones y diferencias de las características intrínsecas y extrínsecas de los alumnos identificados con aptitudes sobresalientes.

Resultados. De los 666 alumnos se encontró que 93 de ellos cumplían con los criterios establecidos en la literatura para ser considerados con aptitudes sobresalientes. Los datos obtenidos permitieron por un lado, establecer el perfil general del grupo y por otro, un perfil más específico, según la agrupación de las características. En ambos casos se pudo apreciar tanto la homogeneidad como heterogeneidad de cada grupo con respecto tanto a la presencia de las características intrínsecas y extrínsecas como a las relaciones entre ellas.

Discusión. Los hallazgos ponen de manifiesto que los adolescentes con aptitudes intelectuales sobresalientes constituyen una población heterogénea, que si bien comparten ciertas características tanto intrínsecas como extrínsecas, difieren en otras, según los niveles de sobresaliencia, lo cual deberá tomarse en cuenta al diseñar programas educativos que puedan cubrir sus necesidades tanto internas como del medio que los rodea, para que puedan desarrollarse exitosamente en los niveles de educación superior.

Palabras claves: sobresaliencia, creatividad, capacidad intelectual, autoconcepto académico.

Recibido: 16/06/12 Aceptación inicial: 22/07/12 Aceptación final: 10/11/12 


\section{Abstract}

Introduction. the current models in the study of giftedness as the Triadic Interdependence defined this as favorable outcome of the interaction between intrinsic factors (intellectual ability, creativity and motivation) and extrinsic (family, peers and school). When considering this, the purpose of this study was to identify and establish a profile of gifted adolescents that include both intrinsic and extrinsic characteristics as well as analyze the relationships and the differences in these characteristics within the group resulting in the profile.

Method. participants were 666 students public school, age ranged between 12 to 18 years with a mean of 14.77. Various statistical analyzes were performed as Pearson's correlation, Student's t test and ANOVA to compare the possible similarities and differences in the intrinsic and extrinsic characteristics the identified gifted students.

Results. Out of 666 students found that 93 of them met the criteria established in the literature to be considered gifted. The data obtained allowed the one hand, establish the general profile of the group and on the other a more specific, according to the grouping of features. In both cases it was possible to determine the homogeneity and heterogeneity of every group with respect to the presence of the intrinsic and extrinsic characteristics as well as relations between them.

Discussion. The discoveries show that although adolescents gifted are a heterogeneous population, which share some intrinsic and extrinsic characteristics, they differ in others, according to giftedness levels, it should taken into account when designing educational programs that can meet your internal needs and of their environment so they can develop successfully in higher education levels.

Keywords: giftedness, creativity, intellectual capacity, academic selfconcept.

Received: 06/16/12 Initial acceptance: 07/22/12 Final acceptance: 11/10/12 


\section{Introducción}

A lo largo de la historia, los sujetos que han manifestado un potencial sobresaliente han destacado en los ámbitos de la cultura, el arte y las ciencias, por lo que han sido ubicados en una posición social especial. No obstante, la conceptuación de este tipo de potencial así como su definición han ido cambiando con el tiempo, en paralelo, han ido evolucionando los modelos que explican las características y los atributos que se deben tomar en cuenta para caracterizar a las personas con aptitudes sobresalientes según las necesidades socioculturales (Monreal, 2000).

Cabe resaltar que ningún modelo ha sustituido a otro, por el contrario, éstos se han ido desarrollando con mayor amplitud y riqueza de información. Se inició con modelos unidimensionales que hacían referencia sólo a la medición del CI hasta los que en la actualidad le dan una importancia no sólo a la parte cognoscitiva, sino también a la afectiva y social. Estos últimos son relevantes en el presente trabajo dado que dichos factores pueden afectar de forma positiva o negativa el desempeño y el potencial de los adolescentes con aptitudes sobresalientes.

En México, a partir de 1986 la Secretaría de Educación Pública (SEP) inició la instrumentación de los modelos educativos para atender a niños con aptitudes sobresalientes a lo largo de la educación primaria con el Proyecto de Atención a Niños y Jóvenes con Capacidades y Aptitudes Sobresalientes ${ }^{1}$ (CAS), que posteriormente se amplió de preescolar hasta la secundaria (SEP, 2006; Zavala y Rodríguez, 2004). No obstante, algunas entidades no lo llevan a cabo, como es el caso del Distrito Federal, el cual identifica a los alumnos sobresalientes a través de un promedio escolar ${ }^{2}$ de 9 o superior (Secretaria de Educación de la Ciudad de México [SECM], 2008). Esto ha generado que los niveles de atención a estos alumnos hayan ido decayendo con el tiempo, de modo que para el ciclo escolar 2007-2008 en educación básica sólo se atendió en total al $0.57 \%$ de niños con aptitudes sobresalientes (SEP, 2008). Con lo anterior, se hace evidente no sólo la limitada detección de los alumnos sobresalientes en los niveles educativos básicos y la nula en los demás niveles, sino también el contraste entre lo que dictan las políticas y lo que sucede en las aulas.

\footnotetext{
${ }^{1}$ Término oficialmente empleado en México para llamar a las personas con altas capacidades o superdotados

${ }^{2}$ En México las calificaciones escolares se puntúan de 0 a 10
} 
En este sentido, la identificación y la caracterización de adolescentes con aptitudes sobresalientes se torna trascendental por varias razones: primero, el Sistema Educativo Mexicano ha orientado sus acciones desde la década de los 80 's hacia la atención a los alumnos sobresalientes en el nivel de educación primaria, pero los pierde cuando éstos pasan a la secundaria (Puga, 2004), lo cual significa que se deje sin atención a estos estudiantes cuando entran en el periodo de la adolescencia, periodo de la vida que de acuerdo a Mönks (2008) puede ser más crítico para los alumnos sobresalientes que para cualquier otra persona, por algunas de sus características como la extremada sensibilidad o el perfeccionismo.

En segundo lugar, las aptitudes sobresalientes no son estáticas y pueden verse afectadas por aspectos como la edad, sexo, el nivel socioeconómico, entre otros (Mönks, 2008; Zacatelco y Acle, 2009; Zavala, 2004), o incluso, pueden estar latentes y no manifestarse (Castelló y Batlle, 1998). Por lo anterior, no siempre se han podido detectar en etapas tempranas del desarrollo, como habría podido ser el caso de Einstein, Freud, Picasso, entre otros, que no brillaron en la infancia sino durante la adolescencia o hasta la adultez (Gardner, 1995). Al respecto, Preckel, Goetz, Pekrun y Klein (2008) encontraron que en Alemania, el 44\% de los estudiantes de entre 11 a 15 años que cursaban el Gymnasium (Instituto de Segunda Enseñanza), no habían sido detectados en etapas tempranas.

Otra razón de importancia para conocer el perfil de los adolescentes sobresalientes es que las investigaciones orientadas al diseño de instrumentos para la identificación de este tipo de alumnos se han centrado sobre todo en la detección de los niños en educación básica (Covarrubias, 2001; Zacatelco, y Acle, 2009; Zavala, 2004) y se ha dejado de lado la validación de instrumentos en población adolescente (Ordaz y Acle, 2010). Por último, la identificación del potencial sobresaliente de los alumnos en esta etapa es relevante tanto para la orientación vocacional futura que se les pueda brindar y que sea acorde a sus intereses, como para el país que requiere del desarrollo de este potencial para la solución de las problemáticas en que se encuentra inmerso (Armenta, 2008; Ordaz y Acle, 2010).

Al tomar en cuenta que el desarrollo de la aptitud sobresaliente es un fenómeno dinámico, dependiente de los cambios del individuo y de su entorno (Mönks, 1996), se considera relevante 
estudiar a los adolescentes que presentan estas aptitudes a través de modelos psicosociales, en particular, el de Interdependencia Triádica de Mönks y Boxtel, pues, los autores en su modelo toman en cuenta este dinamismo, definiendo la aptitud sobresaliente como la interacción favorable entre los factores intrínsecos (habilidad intelectual, creatividad y motivación) y los factores extrínsecos (familia, amigos y escuela). Los factores intrínsecos, a los que se refieren los autores, son: habilidad intelectual excepcional, creatividad y motivación (Mönks y Boxtel, 1985).

La habilidad intelectual toma en cuenta tanto a las habilidades generales como a las específicas (Renzulli, 1978, 1994). Las habilidades intelectuales generales se refiere a la capacidad de procesar información, integrar experiencias que dan como resultado respuestas apropiadas y adaptadas a nuevas situaciones y la capacidad de desarrollar un pensamiento abstracto (Renzulli, 1978, 2002). Este tipo de habilidades se miden a través de las pruebas de inteligencia, considerando en este rubro a aquellas personas con un CI que se ubica de un 5 a un $10 \%$ en el nivel superior de su grupo de referencia (Mönks, 2008). Por otro lado, las habilidades intelectuales específicas: capacidad de adquirir conocimientos, patrones o habilidades para realizar una o más actividades más especializada (Renzulli, 1978, 2002; Mönks, 2008).

La creatividad se define como un proceso que genera sensibilidad a los problemas, deficiencias, grietas o lagunas en el conocimiento y que lleva a identificar dificultades (Mönks, 2008; Torrance, 1962; Torrance, Ball y Safter, 2008). La persona creativa no sólo busca soluciones, sino es aquella que se desvía del camino para encontrar problemas (Getzels, 1981; Guilford, 1967; Landau, 1987). Por último, la motivación implica que una persona se sienta atraída fuertemente por una determinada tarea u objetivo, que le suponga placer trabajar en ello, además, que la persona cuente con una alta capacidad para planificar tanto a corto como a largo plazo (Mönks, 2008).

Basado en los trabajos de Renzulli (1978, 1994), Mönks (2008) esquematiza estos tres factores como tres anillos que se intersectan, y ambos autores señalan, que los sobresalientes son aquellos que poseen o son capaces de desarrollar este conjunto de rasgos, no necesariamente que los presentan (Renzulli, 2002). Este último punto es de gran relevancia por dos razones: Ferrando, Prieto, Ferrándiz y Sánchez (2005) refieren que la inteligencia y la creatividad son capacida- 
des independientes, incluso Simonton (1994) y Sternberg (1996) sugieren que CI muy altos pueden obstaculizar la creatividad, por otra parte, Covarrubias (2001) y Monreal (2000) señalan que algunos individuos de altos logros, como Einstein o Picasso mostraron picos y valles en su creatividad y motivación; esto es, que la creatividad y la motivación fluctúan fácilmente y pasan por periodos de ascenso y descenso, lo que puede dar como consecuencia que algunos métodos de identificación sólo tomen como base el CI considerando que es estático y no se modifica (Covarubias, 2001; Rayo, 1997; Rojo, 1999).

No obstante, algunos autores consideran que la medición del CI por sí solo no puede considerarse como el criterio único para determinar si un estudiante es sobresaliente y refieren que la creatividad es una característica indispensable en su identificación, por lo tanto, es conveniente integrarla en los procesos de detección y evaluación (Castejón, Prieto y López, 2000; Castelló, 1993; Castelló y Batlle, 1998; Genovard y Castelló, 1990; Genovard, Gotzens, Castelló, C. González y P. González, 1992; Guilford, 1977; Landau, 1997; Mönks, 2008; Mönks y Boxtel, 1985; Renzulli, 2002; Torrance, 1984; Zacatelco, 2005; Zavala, 2004). Además, diversas investigaciones han demostrado que otra de las características intrínsecas de los adolescentes con aptitudes sobresalientes es el autoconcepto (Feldhusen, 1986; Mönks, 1996; Boxtel y Mönks,1992), en especial el que se refiere al área académica debido a que incide significativamente en el correcto funcionamiento del ámbito cognoscitivo (González-Pineda, Núñez, González-Pumariega y García, 1997; Mönks, 1996; Santana, Feliciano y Jiménez, 2009; Tannembaum, 1983) e influye directa y substancialmente sobre el aprendizaje (Vidals, 2005), los logros y las expectativas escolares del alumno (Amezcua y Fernández, 2000; Broc, 2000; Henson y Heller, 2000; Möller y Pohlman, 2010; Musitu, García y Gutiérrez, 2002), y esto a su vez, le ayuda a realizar diversas estrategias cognoscitivas y de autorregulación para su aprendizaje escolar (González-Pineda et al., 1997; Monereo, Castello, Clariana, Palma y Pérez, 1994). Por lo anterior, Feldhusen (1986), Mönks y Boxtel (1985) proponen que el autoconcepto se considere como otra de las variables claves para la detección de los alumnos con aptitudes sobresalientes.

El autoconcepto académico es la percepción y evaluación de un estudiante sobre sus habilidades académicas (Cokley, 2000; House, 1992; Marsh y Craven, 2002; Schunk, 1991), esto es, sobre las aprobaciones o reprobaciones de sus expectativas de logros escolares (Herrera, Ramí- 
rez, Roa y Herrera, 2004). El estudio del autoconcepto académico para los adolescentes con aptitudes sobresalientes es relevante debido a diversas razones: los alumnos con aptitud sobresaliente manifiestan un alto autoconcepto académico en comparación al no académico (Boxtel y Mönks, 1992; Hoge y Renzulli, 1993). Así mismo, estos estudiantes muestran un autoconcepto académico más alto en comparación de sus pares no sobresalientes (Boxtel y Mönks, 1992; Galindo, Martínez y Arnáiz, 1999; Herrera et al., 2004; Peñas, 2008).

Por otro lado, no hay diferencias entre el autoconcepto académico de hombres y mujeres sobresalientes (Kelly y Colangelo, 1984). Tampoco se han encontrado diferencias de autoconcepto académico entre diferentes grados --alta, media y moderada-- de aptitud sobresaliente (Brody y Benbow, 1986). Por último, se ha encontrado que este constructo parece mantenerse estable en el tiempo (González-Pineda et al., 1997; Peñas, 2008). Con lo anterior se puede apreciar que el alto autoconcepto académico puede ser un indicador en la identificación y caracterización de los alumnos con aptitudes sobresalientes. Si bien, Gagné (1991), Tannenbaum (1997), Mönks y Boxtel (1985) señalan la importancia de las características intrínsecas, también refieren que las características extrínsecas o ambientales son elementos indispensables que intervienen en el desarrollo psicológico del ser humano, en particular en el desarrollo del potencial sobresaliente. Los factores extrínsecos a los que se refieren Mönks y Boxtel (1985) son: la escuela, la familia y los amigos.

Con respecto a la escuela, López (2003) establece que, en general, la mayoría de los sobresalientes obtiene un buen promedio académico. No obstante, dado que los procesos y recursos de aprendizaje de los sobresalientes pueden diferir ampliamente de los que siguen sus compañeros (Genovard, Gotzens, Badia y Dezcallar, 2010; Genovard, et al., 1992) y al no recibir del sistema educativo los elementos que les apoyen y que les permitan avanzar o enriquecerse con conocimientos adecuados a su nivel, puede producir, en estos alumnos, tendencia hacia la pereza, apatía, abandono intelectual hasta llegar a ser personas en riesgo de fracaso escolar (Acle y Ordaz, 2010; Alonso y Benito, 1996). Por otra parte, Moriana, Alós, Alcalá, Pino, Herruzo y Ruiz (2006) encontraron que los alumnos que practican actividades extraescolares muestran mejor nivel educativo, más competencias y mayores aspiraciones. 
En lo que respecta a la familia, López (2003) sugiere que ésta contribuye en el desarrollo global de la personalidad del individuo, así como de aspectos muy concretos del desarrollo, como son el pensamiento, el lenguaje y los afectos. Asimismo la autora refiere que en el seno familiar sus miembros interaccionan entre sí, estableciendo objetivos y metas, modelos de comportamiento, así como guía, motivación y apoyo mutuo. En las familias de los estudiantes sobresalientes se reportan características comunes. Gross (1993), Silverman y Kearney (1988), Snowden y Christian (1997), Terman (1925), Zacatelco y Acle (2009) observaron que la mayoría de los sujetos fueron hijos únicos o los mayores entre los hermanos y que las familias eran pequeñas, con una media de dos hijos. Por otro lado, se ha encontrado que los padres, en su mayoría, poseían un nivel de estudios superior al de los padres de la población general (Gross, 1993; Gottfried, Eskeles, Bathurst y Wright, 1999; Renzulli y Park, 2000; Silverman y Kearney, 1988; Snowden y Christian, 1997; Terman, 1925) y que las familias exponían a sus hijos a actividades culturales, intelectuales y deportivas (Gross, 1993; Gottfried et al., 1999; Snowden y Christian, 1997). De hecho, Lozano (2003) indica que los factores familiares de mayor influencia en el rendimiento académico y el aprendizaje se refieren la clase social y el clima familiar.

En este mismo sentido, Moriana et al. (2006) refieren que las expectativas de los padres influyen en el éxito escolar, no obstante, los padres de los chicos sobresalientes muestran diferentes expectativas de sus hijos según sea el sexo; por ejemplo, Dickens y Cornell (1993), Olszewski y Yasumoto (1995), Preckel et al. (2008) observaron que muchas adolescentes con altas habilidades académicas muestran un pobre autoconcepto de sus habilidades matemáticas (compensado por otras áreas) y tienen expectativas negativas de sucesos futuros con respecto a esta temática. Una posible explicación de su bajo autoconcepto académico en matemáticas es la influencia paterna adversa, esto es, los autores encontraron que los padres no esperaban que sus hijas fueran tan buenas en matemáticas como lo esperaban de sus hijos varones. Por otra parte, Alonso y Benito (1996) refieren que durante la adolescencia hay un aumento de la separación natural de la familia, siendo la relación con compañeros y amigos más significativa, por lo tanto, las relaciones de amistad le proporcionan la oportunidad de compartir experiencias y saber afrontar los problemas que se presenten. No obstante, Robinson y Olszwski-Kubilius (1997), Rodríguez (2002) y Valadez (2004) encontraron que al entrar a la adolescencia, las mujeres sobresalientes sólo explotan una parte de su potencial y disminuyen sus aspiraciones por el temor al aislamiento o rechazo 
social que, según ellas creen acompañan a los grandes logros. Esto es, que las mujeres sobresalientes conceden mayor relevancia al éxito social que al éxito profesional, su deseo de pertenecer a un grupo es a menudo más fuerte que su voluntad de desarrollarse conforme a sus propias necesidades (Coriat, 1990; Landau, 2003; Peñas, 2008; Reis, 2003, Reis y Renzulli, 2004). Cabe resaltar que Freeman (2004) advierte que lo anterior no pasa necesariamente en todas las culturas, ella encontró en Gran Bretaña que las mujeres adolescentes sobresalientes obtienen mejores logros académicos que los hombres sobresalientes, no sólo en materias sociales sino también en matemáticas y ciencias.

Como se pudo observar son muchas y diversas las características que comparten los sobresalientes, tanto intrínsecas como extrínsecas (Landau, 1997). No obstante, es importante recordar que los alumnos están implicados en múltiples ambientes en los que juegan diversos papeles, en cada uno se espera muestren diversos comportamientos (Kauffman, 1995), por ello, será de capital importancia analizar la forma en que las personas con aptitudes sobresalientes se desenvuelven y se perciben a ellos mismos y a su contexto durante la etapa de la adolescencia para a partir de ahí caracterizar su conducta.

\section{Objetivos}

Al considerar que en México en la educación secundaria y el bachillerato no son detectados los adolescentes con aptitudes sobresalientes y, que como consecuencia, no se cuenta con un perfil de éste tipo de alumnos que permitiera darles el apoyo educativo que requieren sus altas capacidades, el presente trabajo tuvo como propósito establecer un perfil que incluya tanto características intrínsecas como extrínsecas así como analizar las diferencias respecto a la presencia de estas características dentro del grupo de adolescentes con aptitudes sobresalientes.

\section{Método}

\section{Participantes}

La muestra fue no probabilística, estuvo conformada por un total de 666 participantes, 273 hombres (41\%) y 393 mujeres (59\%), con un rango de edad de 12 a 18 años y una media de 14.77, D.E. de 1.769. De los cuales 314 cursaban la secundaria (47.1\%) y 352 el bachillerato 
(52.9\%). Respecto a otros datos ambientales se observó que el 53\% de los participantes refieren desempeñar alguna actividad extraescolar, siendo el deporte el de mayor preferencia. El número de hermanos que reportaron tener fue de cero a siete con una media de dos, el $52 \%$ mencionaron ser el hijo primogénito. El 35\% de las madres y el 37\% de los padres poseían un nivel educativo de bachillerato.

\section{Instrumentos}

El Test de Matrices Progresivas Avanzadas (Raven, 1993). La prueba de Matrices Progresivas Avanzadas o MPA es una prueba no verbal que busca medir capacidad intelectual general. La escala se compone por dos cuadernos separados que presentan, respectivamente, la Serie I (12 problemas) y la Serie II (36 problemas divididos a su vez en tres series). La Serie I se emplea para familiarizar a los sujetos con la naturaleza de los problemas, y entrenarlos en el método de trabajo.

La Serie II se puede utilizar para discriminar entre personas con inteligencia superior, de dos modos posibles: sin límite de tiempo en la evaluación de la capacidad de observación y pensamiento claro, o con límite de tiempo (40 minutos) en la evaluación de la capacidad intelectual. En cada problema se presenta una matriz de diseños abstractos incompletos, que van incrementando progresivamente el grado de dificultad. El objetivo es seleccionar entre ocho opciones de figuras, la opción correcta para completar el diseño tanto vertical como horizontalmente. Para contestar se debe completar analogías y descubrir para cada problema una ley o una serie, a través de la composición y la disposición de los distintos dibujos.

Con respecto a la confiabilidad, Raven, Court y Raven (2008) mencionan que la prueba mostró para los adultos británicos de capacidad intelectual superior al promedio una alta confiabilidad de test-retest de .91 , en relación a la validez de constructo a través de análisis factorial se encontró una saturación g de .82. Por otra parte, en México Méndez-Sánchez y Palacios-Salas (2001) llevaron a cabo un estudio para obtener los baremos de adolescentes y adultos de la ciudad de Aguascalientes, Ags., México, encontrando puntuaciones similares a las reportadas por Raven, Court y Raven (1993). 
Prueba de Pensamiento Creativo de TTCT-Figural (Torrance, 2008a). La prueba fue desarrollada por Torrance y sus colaboradores en 1966, ha sido revisada en diferentes momentos, 1974, 1984, 1990, 1998 y 2008. Consta de dos pruebas independientes, la prueba TTCT-Verbal y la TTCT-Figural, cada una de ellas con dos formas paralelas, A y B que se pueden utilizar para pretest y postest. El presente trabajo utilizó la versión figural en su forma A, el objetivo de ésta es evaluar las producciones creativas a través de la composición de dibujos. Evalúa las siguientes dimensiones de creatividad (Torrance, Ball y Safter, 2008): fluidez, originalidad, elaboración, resistencia al cierre prematuro y abracción de título.

La fluidez se refiere al número de ideas que una persona expresa a través de respuestas interpretables que utilizan el estímulo de manera significativa. La originalidad se basa en la infrecuencia estadística y las respuestas inusuales dentro de una cultura dada. La elaboración es la capacidad de profundizar a detenimiento para consolidar una idea, se observa a través de la cantidad de detalles que el sujeto añade al dibujo con el objetivo de embellecer, mejorar o perfeccionar su idea. La resistencia al cierre prematuro se observa cuando la persona creativa es capaz de mantenerse abierta, tolerar la ambigüedad y retrasar la opinión lo suficiente para permitir los saltos mentales que conllevan a una idea original y que implican la incorporación de toda la información disponible. Por último, la abstracción de títulos es la habilidad para producir títulos abstractos involucra los procesos de pensamiento como análisis, síntesis y organización, es decir, es la habilidad de capturar la esencia de la información involucrada para representar lo más importante. De acuerdo con el manual de Normas Técnicas de TTCT-Figural (Torrance, 2008b) su coeficiente de confiabilidad para el índice de creatividad es de .985 , la confiabilidad para cada uno de los factores es: fluidez .999, elaboración .957, resistencia al cierre prematuro .966, originalidad .974 y por último, la abstracción de títulos tuvo una confiabilidad de .967.

Escala de Autoconcepto Académico (Ordaz, Reyes-Lagunes y Acle, 2009). Su propósito es identificar niveles altos y bajos de autoconcepto académico, conformada por 28 reactivos, con un Alfa de Cronbach de 0.897 distribuidos en 5 factores: creatividad, motivación, habilidades intelectuales generales, perfeccionismo y habilidades intelectuales específicas. La escala cuenta con cinco opciones de respuestas que iban desde "Nunca" marcada con el número uno, hasta "Siempre" marcada con el número cinco. Se parte de la base de que el sujeto que responda en un 
sentido afirmativo a los enunciados de la escala, tenderá a ser un sujeto que muestre un alto autoconcepto académico en las áreas mencionadas y, por el contrario, si un sujeto responde en sentido negativo hacia los enunciados, tendrá un bajo autoconcepto académico. La ficha de identificación contiene 20 preguntas orientadas a obtener información acerca de datos extrínsecos tales como la posición que tiene el adolescente entre sus hermanos, escolaridad de los padres, su situación socio-económica y las áreas donde emplean sus aptitudes (danza, lenguaje escrito, liderazgo, matemáticas, dibujo, lenguaje oral, memorial, deporte, relaciones sociales y música).

\section{Procedimiento}

La muestra fue tomada de una escuela pública perteneciente a la Universidad Nacional Autónoma de México, la cual brinda secundaria y bachillerato en las mismas instalaciones, cuya población se conforma principalmente por hijos de trabajadores y académicos de dicha institución, además es importante hacer algunas acotaciones sobre las características de la escuela: 1) el criterio de ingreso a la secundaria es tener alto promedio en la primaria; 2) la mayoría de los alumnos de bachillerato ingresan por examen general de conocimientos; 3) los alumnos que ingresan ya sea en la secundaria o bachillerato tienen pase automático a la educación universitaria; 4) la población es heterogénea con respecto a su lugar de procedencia, dado que los alumnos provienen de los diferentes puntos del área metropolitana, esto es de las diversas delegaciones del Distrito Federal y algunos municipios del Estado de México.

Para la aplicación de los instrumentos se solicitó el consentimiento informado tanto de las autoridades de la escuela, como de los maestros de los grupos así como de los participantes quienes se encontraban en su salón de clases con su profesor de asignatura. La aplicación se llevó a cabo en dos sesiones contrabalanceadas. Al iniciar se les proporcionó una breve explicación sobre el propósito y la importancia de contestar los cuestionarios y se les solicitó su aceptación. En la primera sesión se les entregaron los dos cuadernillos de la Serie I y II de la Escala Avanzada de MPA así como la hoja de respuestas, posteriormente se siguieron las indicaciones dadas por el Manual de Test de Matrices Progresivas Escala Avanzada. Al terminar los 40 minutos se les pidió que contestaran la Escala de Autoconcepto Académico la cual duró aproximadamente 15 minutos. 
En la segunda sesión se les volvió a dar una breve explicación sobre el propósito de contestar los cuestionarios y posteriormente se les entregó el cuadernillo de la prueba TTCT-Figural que consta de tres actividades: construcción de dibujos, terminando los dibujos y por último, líneas. En todo momento se siguieron las normas de aplicación de la prueba de Torrance (2008), ofreciendo instrucciones precisas y considerando, como señala el autor, los tiempos de aplicación para cada subprueba, esto fue, 10 minutos para cada actividad un total de 30 minutos. El clima psicológico, antes y durante la administración de la prueba se trató que fuera confortable y estimulante.

\section{Análisis Estadísticos}

Se capturaron, evaluaron y analizaron estadísticamente los datos arrojados con el programa SPSS 19. Con la finalidad de identificar a los adolescentes sobresalientes, se consideraron las puntuaciones mínimas y máximas obtenidas por la población, así mismo se consideró que la detección debe estar basada en un concepto amplio y flexible que dé la oportunidad de identificar una amplia diversidad de sujetos con diferentes características culturales, sociales y raciales $(\mathrm{Ca}-$ llahan, Hunsaker, Adams, More y Bland, 1995; Castelló y Batlle, 1998; Frasier y Passow, 1994; Richert, 1997 y evitar excluir incorrectamente a alumnos (Richert, 1997; Tourón, Peralta y Repáraz, 1998), por ello, se tomó como punto de corte el percentil 75 (Castelló y Batlle, 1998; Martínez y Castelló, 2004; Zacatelco y Acle, 2009) para la capacidad intelectual y, en por lo menos una de las otras dos características intrínsecas.

Dado que los recursos intelectuales de cada individuo son diversos, Armenta (2008), Castelló y Batlle (1998) proponen la utilización de diferentes perfiles. Para establecer el perfil de los adolescentes con aptitudes sobresalientes, en el presente trabajo, se realizaron los siguientes análisis estadísticos, divididos en dos apartados principalmente:

En el primer apartado se presenta un perfil general del adolescente con aptitudes sobresalientes. Para conocer el grado de asociación entre las características intrínsecas y extrínsecas se realizó correlación de Pearson. Posteriormente se determinaron las diferencias por sexo con la prueba t de Student, así mismo para explorar la influencia de la interacción entre las variables sexo y nivel educativo (secundaria y bachillerato) con respecto a las características intrínsecas y extrínsecas se realizó un ANOVA de dos vías. 
En el segundo apartado se presenta un perfil por grupo. Se formaron tres grupos, según las áreas en las que obtuvieron puntuaciones arriba del percentil 75. Posteriormente se utilizó la correlación de Pearson para conocer el grado de asociación entre las características intrínsecas y extrínsecas de cada grupo. Para determinar las diferencias de las características intrínsecas y extrínsecas con respecto a su grupo de pertenencia se realizó un ANOVA de un vía. Por último, se realizó un ANOVA de dos vías con la finalidad de conocer las diferencias por sexo según su grupo de pertenencia con respecto a los datos intrínsecos y extrínsecos.

\section{Resultados}

Con la finalidad de caracterizar a los adolescentes con aptitudes sobresalientes se consideraron las puntuaciones mínimas y máximas de los tres instrumentos aplicados: Matrices Progresivas Avanzadas (MPA), Prueba de Pensamiento Creativo de Torrance (TTCT) y la Escala de Autoconcepto Académico (EAA), dichas puntuaciones se proporcionaban según su grupo de referencia, el cual estuvo dado por la edad. Con base en los criterios establecidos, puntuación arriba del percentil 75 en capacidad intelectual y en por lo menos uno de los otros dos criterios, de 666 alumnos se encontró que 93 alumnos cumplían con dicho criterio para ser considerados con aptitudes sobresalientes. Los 93 alumnos representaron el 14\% de la muestra, de los cuales, 47 fueron hombres y 46 mujeres.

Para describir el perfil general de adolescentes con aptitudes sobresalientes se determinaron las relaciones entre los tres instrumentos aplicados, para ello se llevó a cabo una correlación de Pearson, la cual mostró por un lado, una única asociación y esta fue negativa entre la prueba de creatividad y la de autoconcepto académico $(r=-.495, p<0.01)$. Por otro lado, no se observaron asociaciones entre las características intrínsecas y las extrínsecas, es decir, no se relacionaron el nivel de capacidad intelectual, la creatividad ni el autoconcepto académico con la posición de hijo que ocupa, escolaridad de la madre, escolaridad del padre, trabajo o actividad extraescolar.

En cuanto a las características extrínsecas se observó, en relación a la escuela, que los adolescentes con aptitudes sobresalientes obtienen un promedio escolar de 8.89, el 59\% de ellos realiza una actividad extraescolar que en general es la práctica de algún deporte, por otra parte el área donde mencionaron orientar con mayor facilidad sus aptitudes fue en música y donde se les 
dificulta fue en danza; con respecto al ámbito familiar, se observó que el 55\% es primogénito y que el $46 \%$ de las madres y el $51 \%$ de los padres tienen licenciatura o superior.

Por otro lado, para conocer si existían diferencias estadísticamente significativas entre pares, hombres y mujeres con aptitudes sobresalientes, se realizó la prueba $t$ de Student mediante la cual no se encontraron diferencias significativas $(t=\mathrm{p}<.05)$ con respecto a las puntuaciones medias del nivel de capacidad intelectual, creatividad ni en autoconcepto académico, de la misma forma no se encontraron diferencias con respecto a las características extrínsecas. Para explorar la influencia de la interacción entre las variables sexo (hombre, mujer) y nivel educativo (secundaria y bachillerato) se realizó un ANOVA de dos vías, a través de este análisis no se encontraron diferencias en nivel de capacidad intelectual $(F(1,89)=.529, p>.05)$, la creatividad $(F(1,89)=$ $.963, p>.05)$ ni con el autoconcepto académico $(F(1,89)=.556, p>.05)$, este mismo resultado se corroboró haciendo el análisis con grupos del mismo tamaño.

Por otra parte, con la finalidad de profundizar en el perfil de los adolescentes identificados con aptitudes sobresalientes se conformaron tres grupos, a través de sus características intrínsecas, en concordancia con Armenta (2008), Castelló y Batlle (1998) quienes refieren que las características y necesidades son diferentes según el grado y el tipo de habilidades que presentan, por lo tanto los grupos estuvieron formados de la siguiente forma: el grupo I estuvo formado por 13 alumnos que obtuvieron percentil mayor a 75 en los tres instrumentos, es decir, capacidad intelectual, creatividad y autoconcepto académico. El grupo II conformado por 31 alumnos que obtuvieron percentil mayor a 75 en nivel de capacidad intelectual y creatividad. Por último, el grupo III estuvo integrado por 49 alumnos que obtuvieron percentiles mayores a 75 en el nivel de capacidad intelectual y autoconcepto académico. De esta manera en la Tabla 1 se pueden apreciar las características intrínsecas de cada grupo y el porcentaje en que se ubica. 
Tabla 1. Características de los adolescentes con aptitudes sobresalientes por grupo

\begin{tabular}{|c|c|c|c|c|c|}
\hline \multirow{2}{*}{ Grupos } & \multirow{2}{*}{ Sexo } & \multicolumn{2}{|c|}{ Nivel Educativo } & \multirow{2}{*}{ Total } & \multirow{2}{*}{$\begin{array}{c}\% \text { con } \\
\text { respecto a la } \\
\text { muestra }\end{array}$} \\
\hline & & Secundaria & Bachillerato & & \\
\hline \multirow{3}{*}{$\begin{array}{l}\text { Grupo Uno: } \\
\text { Capacidad intelectual, } \\
\text { creatividad y } \\
\text { autoconcepto académico }\end{array}$} & Masculino & 4 & 5 & 9 & \\
\hline & Femenino & 1 & 3 & 4 & \\
\hline & Total & 5 & 8 & 13 & $2 \%$ \\
\hline \multirow{3}{*}{$\begin{array}{l}\text { Grupo Dos: } \\
\text { Capacidad intelectual y } \\
\text { creatividad }\end{array}$} & Masculino & 5 & 9 & 14 & \multirow{3}{*}{$5 \%$} \\
\hline & Femenino & 7 & 10 & 17 & \\
\hline & Total & 12 & 19 & 31 & \\
\hline \multirow{3}{*}{\multicolumn{2}{|c|}{\begin{tabular}{ll} 
Grupo Tres: & Masculino \\
Capacidad intelectual y & Femenino \\
\multicolumn{2}{l}{ autoconcepto académico Total }
\end{tabular}}} & 11 & 13 & 24 & \\
\hline & & 8 & 17 & 25 & $7 \%$ \\
\hline & & 19 & 30 & 49 & \\
\hline \multirow[t]{4}{*}{ Total } & Masculino & 20 & 27 & 47 & \\
\hline & Femenino & 16 & 30 & 46 & $14 \%$ \\
\hline & Total & 36 & 57 & 93 & \\
\hline & & $5 \%$ & $9 \%$ & $14 \%$ & \\
\hline
\end{tabular}

Es interesante observar que los alumnos del grupo I representan al 2\% de la población sobresaliente coincidiendo con la incidencia que refiere el Organismo Mundial de la Salud (OMS) y Terman (1925). Con la finalidad de conocer cómo se asociaban las características intrínsecas y extrínsecas dependiendo del grupo de referencia, se realizó un análisis de correlación de Pearson para cada uno de los tres grupos, encontrando que:

Para el grupo I se apreció una asociación estadísticamente significativa y negativa entre el nivel de capacidad intelectual y creatividad $(r=-.612, p<0.05)$. Así mismo, se encontraron relaciones entre las áreas y los factores. El nivel de capacidad intelectual se asoció con algunos factores de creatividad, se relacionó negativamente con fluidez $(r=-.598, p<0.05)$, originalidad $(r=-$ $.559, p<0.05)$, y resistencia al cierre $(r=-.678, p<0.05)$, esto era esperado dado que la capacidad intelectual procesa información para encontrar una respuesta apropiada, en comparación de fluidez que busca diferentes respuestas; con originalidad se contrapone debido a que éste busca una respuesta inusual que no necesariamente es correcta; por último, contrastó también con resis- 
tencia al cierre debido a que éste se trata de mantenerse abierto a nuevas ideas, tolerar la ambigüedad, aplazar la opinión, en cambio la capacidad intelectual trata de cerrar a través de encontrar la respuesta correcta. Por otro lado, el autoconcepto académico se relacionó positivamente con el factor elaboración de creatividad $(r=.581, p<0.05)$, esto es, entre mayor capacidad de profundizar o perfeccionar una idea el alumno se percibe con mayor habilidad académica.

Entre las características extrínsecas se observó, en el ámbito escolar, que este grupo obtuvo un promedio escolar de 9.30, que el 77\% de los alumnos realizaba alguna actividad extraescolar, las áreas donde orientan sus capacidades son matemáticas y deportes. Con respecto al ámbito familiar se apreció que el $62 \%$ era hijo único o primogénito, el $69 \%$ tanto de madres como de padres poseían estudios superiores; el $31 \%$ restante poseía estudios técnicos o de nivel medio superior.

En el grupo II se advirtió una asociación significativa y positiva entre creatividad y autoconcepto académico $(r=.441, p<0.05)$. Asimismo se observaron correlaciones entre el nivel de capacidad intelectual y los factores de creatividad, las asociaciones positivas fueron de capacidad intelectual con abstracción de títulos $(r=.409, p<0.05)$, y con habilidad intelectual general $(r=$ $.468, p<0.05)$; de forma negativa con resistencia al cierre $(r=-.484, p<0.01)$ y con habilidad intelectual específica $(r=-.484, p<0.01)$. Por otra parte, autoconcepto académico correlacionó con el factor originalidad de la prueba de creatividad $(r=.367, p<0.05)$.

Entre las características extrínsecas se observó, en el ámbito escolar, que este grupo obtuvo un promedio escolar de 8.80 y que el $58 \%$ de los alumnos realizaba alguna actividad extraescolar, el área donde mencionaron orientar sus capacidades fue en deportes. Con respecto al ámbito familiar se encontró que el $48 \%$ era hijo único o primogénito, el $39 \%$ de madres y el $40 \%$ padres tenían estudios superiores; el 31\% de madres y el 26\% de padres sólo tenían educación básica (primaria y secundaria).

En el grupo III únicamente hubo una asociación estadísticamente significativa, entre el nivel de capacidad intelectual y elaboración de la prueba de creatividad $(r=.412, p<0.01)$. Este grupo obtuvo un promedio escolar de 8.86 y el 55\% de los alumnos realizaba alguna actividad 
extraescolar, el área donde mencionaron orientar sus capacidades fue en memoria. Con respecto al ámbito familiar se apreció que el $62 \%$ era hijo único o primogénito, el $43 \%$ de madres y el $52 \%$ padres tenían estudios superiores; el 22\% de madres y el 16\% de padres sólo tenían educación básica.

Con el objetivo de analizar las diferencias entre los grupos con respecto a las características intrínsecas, se realizó un ANOVA de una vía el cual arrojó sólo las diferencias esperadas; esto es, se observa que: a) no hubo diferencias entre los grupos sobre las medias de capacidad intelectual $(F(2,90)=1.249, p>.05)$; b) con respecto a creatividad si hubieron diferencias entre los tres grupos $(F(2,90)=95.083, p<.01)$, el análisis posterior con la prueba de Scheffé señaló que el grupo I $\left(M_{I}=80.46\right)$ y II $\left(M_{I I}=80.03\right)$ obtuvieron mayor puntuación en comparación con el grupo III $\left(M_{I I I}=49.65\right)$; c) en autoconcepto académico $(F(2,90)=143.582, p<.01)$, el análisis posterior con la prueba de Scheffé señaló que el grupo I $\left(M_{I}=115.92\right)$ y III $\left(M_{I I I}=115.41\right)$ obtuvieron mayor puntuación en comparación con el grupo II $\left(M_{I I}=86.81\right)$. Es importante señalar que las diferencias fueron esperadas por la propia naturaleza de la formación de los grupos.

Con respecto a los datos extrínsecos, se encontraron diferencias estadísticamente significativas en escolaridad de la madre $(F(2,67.19)=3.371, \mathrm{p}<.05)$, a través de la prueba de GamesHowell se observó que las madres de los alumnos del grupo I $\left(M_{I}=4.69\right)$ presentaban mayor escolaridad que las madres de los alumnos del grupo II $\left(M_{I I}=3.77\right)$ y Tres $\left(M_{I I I}=3.79\right)$. Así mismo se encontraron diferencias en escolaridad del padre $(F(2,70.71)=3.971, p<.05)$, a través de la prueba de Games-Howell se advirtió que los padres de los alumnos del grupo I $\left(M_{I}=4.92\right)$ presentaban mayor nivel de escolaridad que los padres de los alumnos del grupo II $\left(M_{I I}=3.78\right)$.

Por otro lado, se apreció que el grupo I obtuvieron un promedio escolar de 9.30 mayor que los grupos II y III (8.80 y 8.86 respectivamente), no obstante, las diferencias no fueron estadísticamente significativas $(F(2,71)=1.263, p>.05)$, así mismo no se encontraron diferencias entre los grupos respecto a la posición de hijo $(F(2,90)=.654, p>.05)$, ni al realizar una actividad extraescolar $(F(2,90)=.244, p>.05)$, tampoco en los promedios ni de educación primaria $(F(2,84)=1.252, p>.05)$ ni secundaria $(F(2,52.10)=2.449, p>.05)$. 
También se pudo apreciar que las áreas de interés donde orientan sus aptitudes fueron diferentes dependiendo del grupo que pertenecen, esto es, el grupo I refirió poseer mayor destreza en matemáticas y en deportes; los alumnos del grupo II en deportes; los alumnos del grupo III en memoria y relaciones sociales. Por otra parte, los adolescentes del grupo I mencionaron tener menor destreza en dibujo y el grupo II y III refirieron menor destreza en danza.

Para determinar la diferencia entre los tres grupos con respecto a las áreas donde orientan sus habilidades se realizó un ANOVA de una vía en el cual sólo se encontraron diferencias estadísticamente significativas en el área de memoria $(F(2,88)=3.087, p<.05)$, a través de la prueba de Scheffé se observó que los alumnos del grupo I $\left(M_{I}=5.00\right)$ y III $\left(M_{I I I}=4.60\right)$ mencionaron tener mejor memoria en comparación con los del grupo II $\left(M_{I I}=6.23\right)$, se recuerda que a menor puntuación significa mayor destreza.

Por último, para conocer si existían diferencias estadísticamente significativas entre sus pares, esto es entre hombres y mujeres con aptitudes sobresalientes según su grupo de pertenencia, con respecto a las características intrínsecas se realizó un ANOVA de dos vías. No se encontraron diferencias para el nivel de capacidad intelectual, ni para autoconcepto académico. Por otro lado, se encontró influencia de la interacción entre el sexo y los grupos con respecto a la creatividad $(F(2,87)=5.130, p<.01)$, a través de la prueba de Scheffé se observó que las diferencias se encuentran en el grupo III, y muestran más creativas a las mujeres que a los hombres, este mismo resultado se obtuvo con grupos del mismo tamaño.

\section{Discusión y conclusiones}

El propósito del presente trabajo fue establecer el perfil de alumnos con aptitudes sobresalientes de un bachillerato perteneciente a la UNAM. Es trascendental referir como ya se señaló, que, a nivel oficial, en México no ha habido suficiente atención a todos los alumnos que presentan capacidades sobresalientes ni un seguimiento de aquellos que han sido atendidos en el nivel de educación primaria (Puga, 2004); esto es, se han dejado sin atención, principalmente, a estudiantes adolescentes. Cortés (2010) hace énfasis además en aquellas políticas instrumentadas que han estado desarticuladas, con un presupuesto raquítico que reflejan la ausencia de una política 
real y por ende, existen algunos modelos que no han logrado los resultados tangibles esperados, como es el caso particular del proyecto de atención a niños y jóvenes con capacidades y aptitudes sobresalientes. De aquí, surge la necesidad de establecer el perfil de estudiantes sobresalientes durante la adolescencia, periodo crítico del desarrollo, con objeto de detectarlos, promover su potencial, darle una orientación vocacional acorde a sus aptitudes, así como el apoyo emocional que le de soporte a su extremada sensibilidad y elevado perfeccionismo.

Dicha identificación se fundamentó en el modelo de Interdependencia Triádica (Mönks y Boxtel, 1985), debido a que en él se establece la importancia tanto de las características intrínsecas como extrínsecas y de las relaciones que provienen de ellas, lo cual permite obtener una visión integral de la interacción de dichas características en la presencia de las aptitudes sobresalientes de la muestra de estudio, que en este caso se refiere a un bachillerato público al que asiste población de diferentes niveles socioeconómicos y educativos. Con base en lo anterior, los resultados permitieron establecer, en un primer momento, el perfil general, y posteriormente, caracterizar de manera más amplia un perfil diferencial que dio cuenta de la heterogeneidad al interior del grupo general.

En relación al perfil general los resultados revelan que el 14\% de la muestra se ubicó con un perfil sobresaliente, lo cual indica una alta incidencia y da cuenta de una importante población que no está siendo considerada en las estadísticas y programas oficiales. De este perfil general se observaron aspectos interesantes tanto de los factores intrínsecos como de los factores extrínsecos. Con respecto a los intrínsecos se observó que la creatividad y autoconcepto académico se relacionaron negativamente, lo que indica que cuando los alumnos obtenían altos niveles de creatividad en contraposición mostraban bajos niveles de autoconcepto académico, una posible explicación puede darse con base en la investigación de Landau (1997) quien refiere que las personas con altos índices de creatividad no siempre son bien vistos por los profesores y ello puede afectar tanto al rendimiento como al autoconcepto académico.

En cuanto a los factores extrínsecos encontrados para el perfil general, en relación a la escuela, se apreció que la mayoría de los alumnos sobresalientes saca buenas calificaciones como menciona López (2003), no obstante, también hubieron algunos de ellos con bajas notas, con 
promedio de 6, por lo tanto, utilizar el promedio como único criterio de identificación no es suficiente, como sucede en el Programa de Niños Talento (SECM, 2008), ya que puede excluir a muchos alumnos que no presentan notas altas. Otra característica extrínseca importante advertida de estos alumnos, fue que la mayoría realizaba alguna actividad extraescolar, siendo el deporte la de mayor preferencia. Con respecto al ámbito familiar, se observó que el 55\% de los sobresalientes eran primogénitos, esto coincide con lo encontrado por Gross (1993), Silverman y Kearney (1988), López (2003), Terman (1925), Zacatelco y Acle (2009).

Así mismo, se encontró que el $69 \%$ de los padres habían realizado estudios universitarios o de posgrado concordando con lo señalado por Gross (1993), Gottfried et al. (1999), López (2003), Silverman y Kearney (1988) y Terman (1925) quienes refieren que la mayoría de los padres de los sobresalientes tienen altos niveles académicos. No obstante, al contrario de lo antes señalado un hallazgo relevante de este estudio es que hubo un alto porcentaje de padres de los adolescentes identificados como sobresalientes que presentaban bajos niveles educativos, con lo anterior una vertiente sería analizar más sobre las expectativas paternas y cohesión familiar que han permitido desarrollar las aptitudes de estos alumnos.

Es importante mencionar que en el perfil general sólo se encontraron diferencias entre hombres y mujeres en relación al porcentaje de identificados, no hubieron diferencias en sus puntajes sobre características intrínsecas ni extrínsecas, esto es, hombres y mujeres, puntuaron igual en capacidad intelectual, creatividad y autoconcepto académico; así mismo, el contexto social en el que se desenvolvían fue parecido. Lo cual difiere a los hallazgos de Peñas (2008), Robinson y Olszwski-Kubilius (1997), Rodríguez (2002) y Valadez (2004) que encontraron que en la adolescencia las mujeres disminuyen sus aspiraciones y sus logros académicos. Probablemente las semejanzas encontradas entre las características de hombres y mujeres se deban a las expectativas que tienen los padres sobre sus hijos, pues ellos saben que al entrar a esta escuela en particular, el alumno tiene garantizado su ingreso a la universidad. Por lo tanto, una línea de futura investigación podría ser estudiar el efecto que tienen las expectativas paternas sobre la actuación académica de sus hijos sobresalientes. 
Se mencionó anteriormente que las personas con aptitudes sobresalientes no constituyen una población con características homogéneas (Mönks, 2008; SEP, 2006; Zacatelco y Acle, 2009; Zavala, 2004) asimismo se señaló sobre la conveniencia de diferenciar los grados de sobresaliencia (Armenta, 2008; Castelló y Batlle, 1998) debido que, tanto las características como el apoyo que requieren son diferentes según las habilidades y el grado que presenten, de aquí la importancia de diferenciar a los alumnos identificados a través de los diferentes niveles obtenidos en los instrumentos aplicados, el análisis de éstos dio como resultado tres perfiles con interesantes características cada uno, que a continuación se comentan.

Para iniciar se pudo observar que los alumnos del grupo I mostraron a mayor capacidad intelectual menor creatividad, esta relación fue interesante dado que Ferrando et al. (2005) y Landau $(1987,2003)$ señalan que una gran inteligencia no condiciona la creatividad. Al respecto Torrance (1962) postula, a través de su Teoría del Umbral, que cuando el CI está por debajo de cierto límite, la creatividad también se encuentra limitada, mientras que cuando el CI se sitúa por encima de ese límite $(\mathrm{CI}=115-120)$ la creatividad llega a ser una dimensión casi independiente. Por su parte, Zavala (2004) refiere que la Teoría del Umbral abrió la posibilidad de considerar que los sujetos inteligentes carecieran de características creativas elevadas, de hecho, Simonton (1994) y Sternberg (1996) sugieren que CI muy altos pueden obstaculizar la creatividad.

En lo que respecta al ámbito escolar se pudo observar que si bien los tres grupos tenían promedios por arriba del 8.80 el grupo I tuvo las calificaciones más alta, confirmando que la mayoría de los sobresalientes saca buenas calificaciones en la escuela (López, 2003), pero además, Moriana et al. (2006) señalan que los alumnos obtienen mejor rendimiento académico cuando realizan actividades extraescolares, al respecto se observó que la proporción de alumnos del grupo I que realizaban una actividad fuera de la escuela fue un $20 \%$ superior con respecto a los otros dos grupos, al considerar que los padres de este grupo presentan los más altos niveles educativos en comparación con los otros dos grupos coincide con lo señalado por López (2003) que los padres con mayores estudios proporcionan a sus hijos mayores actividades tanto culturales como deportivas. 
Por su parte, el grupo II mostró alta capacidad intelectual y alta creatividad sin embargo manifestó un bajo autoconcepto académico, éste último se vio reflejado en su rendimiento académico, que a pesar de no haber diferencias estadísticamente significativas, se pudo observar que fue el grupo con promedio escolar más bajo, esto probablemente sea debido a que el autoconcepto académico influye sobre los logros y las expectativas escolares del alumno (Amezcua y Fernández, 2000; Broc, 2000; Henson y Heller, 2000; Möller y Pohlman, 2010; Musitu, García y Gutiérrez, 2002), así como al desarrollo de estrategias cognoscitivas y de autorregulación para su aprendizaje escolar (González-Pineda et al., 1997; Monereo, Castello, Clariana, Palma y Pérez, 1994). Otra posible explicación es que los padres de este grupo son los que tienen el nivel educativo más bajo, lo que origina que no puedan darles el soporte educativo necesario (Gómez y Valadez; 2010; López, 2003; Renzulli y Park, 2000). Adicionalmente es importante mencionar que aquellos sobresalientes con bajo autoconcepto académico y con padres de bajos niveles educativos están en riesgo de fracaso escolar (López, 2003; Acle y Ordaz, 2010), por lo tanto, sería importante que se les proporcionara un soporte adicional, tanto académico como emocional.

Los alumnos del grupo III obtuvieron alta capacidad intelectual y alto autoconcepto académico, a pesar de ser el grupo con baja puntuación en creatividad, no hubo ninguna diferencia, con respecto a los otros dos grupos, en la manera que elaboraron sus diseños, es decir, estos alumnos mostraron la misma capacidad de detallar, profundizar, mejorar y perfeccionar sus ideas. Por otro lado, fue interesante observar que únicamente en este grupo se presentaron diferencias por sexo, se apreció que las mujeres mostraron un mayor índice de creatividad, por ello se puede concluir que este grupo en particular difiere de lo señalado por Coriat (1990), Landau (2003), Peñas (2008), Robinson y Olszwski-Kubilius (1997), Rodríguez (2002) y Valadez (2004), quienes en sus estudios encontraron que las mujeres adolescentes sobresalientes sólo explotaban una parte de su potencial y disminuyen sus aspiraciones por el temor al aislamiento o rechazo social, por lo que se corrobora lo señalado por Freeman (2004) respecto que las diferencias por sexo no necesariamente se presentan en todas las culturas.

De esta manera, los hallazgos ponen de manifiesto que los adolescentes con aptitudes intelectuales sobresalientes constituyen una población heterogénea, que si bien comparten ciertas características tanto intrínsecas como extrínsecas, difieren en otras, según los niveles de sobresa- 
liencia (Armenta, 2008; Castelló y Batlle, 1998), lo cual deberá tomarse en cuenta al diseñar programas educativos que puedan cubrir sus necesidades tanto internas como del medio que los rodea, para que puedan desarrollarse exitosamente en los niveles de educación superior.

Por último, en relación a las limitaciones del estudio, es preciso destacar que el diseño de la investigación sólo permitió identificar las características de contexto que el sobresaliente refirió, no obstante, sería importante conocer con mayor profundidad los actores que rodean su contexto social, es decir, familia y escuela. Se sabe que no sólo las características de las familias constituyen un elemento que influye en el desempeño del sobresaliente, sino también la percepción, las expectativas y la implicación académica que los padres tienen de sus hijos son factores determinantes. Por otro lado, al observar el contexto escolar sólo se incluyó el rendimiento académico, sin embargo, hay dos actores que influyen en este rendimiento: los profesores y los amigos. Es trascendental conocer la opinión que el profesor tiene respecto al tema, así como su percepción y expectativas al respecto, ya que ellos pueden ser una guía y motivarlos para su crecimiento intelectual o ser un obstáculo y desmotivarlos. Otro actor importantísimo para los estos adolescentes son sus amigos y compañeros de clase, debido a que éstos influyen en la actuación del sobresaliente dada su necesidad de pertenencia. Por otra parte, debido al localismo de la muestra, se hace necesario replicar esta investigación en otras escuelas de nivel medio superior.

\section{Referencias}

Acle, T. G. y Ordaz, V. G. (2010). Resiliencia y aptitudes sobresalientes en niños de zonas urbano-marginadas. Ideacción (31), 288-299.

Alonso, J. y Benito, Y. (1996). Superdotados: Adaptación Escolar y Social en Secundaria. España: Narcea.

Amezcua, J. y Fernández, E. (2000). La influencia del autoconcepto en el rendimiento académico. Iberpsicología, 5(1). Recuperado de:

http://www.fedap.es/IberPsicologia/iberpsi5-1/amezcua/amezcua.htm

Armenta, C. (2008). Educación incluyente para sobresalientes en las sociedades del conocimiento. Revista Internacional de Ciencias Sociales y Humanidades, 18(1), 109131. 
Boxtel, H. W. y Mönks, F. (1992). General. social, and academic self-concepts of gifted adolescents. Journal of Youth and Adolescence, 21(2), 169-186.

Broc, M. (2000). Autoconcepto, autoestima y rendimiento académico en alumnos de $4^{\circ}$ de la E.S.O. Implicaciones psicopedagógicas en la orientación y tutoría. Revista de Investigación Educativa, 18(1), 119-146.

Brody, L. y Benbow, C. (1986). Social and emotional adjustemet of adolescents extremely talented in verbal or mathematical reasoning. Journal of Youth and Adolescence, 15, 1-18.

Callahan, C. M., Hunsaker, S. L., Adams, C. M. More, S. D. y Bland, L. C. (1995). Instruments used in the identification of gifted and talented students. Storrs, CT: Monografía de investigación no. 95130 del The National Research Center on the Gifted and Talented, University of Connecticut.

Castejón, J. L., Prieto, M. D. y López, O. (2000). Evaluación y desarrollo de la creatividad. En M. D. Prieto y J. L. Castejón. (Eds.), Los Superdotados. Esos alumnos excepcionales (pp. 77-111). Málaga: Aljibe.

Castejón, J. L., Prieto, S. y Rojo, M. (1999). Modelos y estrategias de identificación del superdotado. En D. Prieto (Ed.), Identificación, evaluación y atención a la diversidad del superdotado (pp. 17 -40). Málaga, España: ALJIBE.

Castelló, A. (1993). Creatividad. En L. Pérez (Ed.), 10 palabras claves en superdotados. Navarra: Verbo Divino.

Castelló, A. y Batlle, C. (1998). Aspectos teóricos e instrumentales en la identificación del alumno superdotado y talentoso. Propuesta de un protocolo. FAISCA, 6, 26-66.

Cokley, K. (2000). An investigation of academic self-concept and its relationship to academic achievement in African American college students. Journal of Black Psychology, 26, 148-64.

Coriat, A. (1990). Los niños Superdotados: Enfoque psicodinámico y teórico. Barcelona: Herder.

Cortés, C. L. (2010). Hacía una política pública de educación de talentos. El caso de México. (Tesis de doctorado en Políticas Públicas Comparadas inédita). Facultad Latinoamericana de Ciencias Sociales: México.

Covarrubias, P. (2001). Características cognitivas y socioafectivas de los niños y niñas sobresalientes de la zona norte de México (Tesis de Maestría en Educación Especial). México: Universidad Autónoma de Tlaxcala. 
Dickens, M. y Cornell, D. (1993). Parent influences on the mathematics self-Concept of high ability adolescent girls. Journal for the Education of the Gifted, 17(1), 53-73.

Feldhusen, J. F. (1986). A conception of giftedness. En R. J. Sternberg y J. Davidson (Eds.), Conceptions of Giftedness (pp. 112 - 127). Cambridge: Cambridge University Press.

Ferrando, Prieto, Ferrándiz y Sánchez (2005). Inteligencia y Creatividad. Electronic Journal of research in educational psychology, 3(3), 21-50.

Frasier, J. y Passow, A. H. (1994). Toward a new paradigm for identifying talent potential. Storrs, CT. USA: Monografía de investigación no. 94112 del National Research Center on the Gifted and Talented.

Freeman, J. (2004). Cultural influences on gifted gender achievement. High Ability Studies, 15(1), 7-23.

Gagné, F. (1991). Towards a differentiated model of giftedness and talent. En N. Colangelo y G. A. Davis (Eds.), Handbook of gifted education (pp. 65 - 80). Boston: Allyn and Bacon.

Galindo, A., Martínez, F. y Arnáiz, P. (1999). El autoconcepto en el superdotado. En D. Prieto (Ed.), Identificación, evaluación y atención a la diversidad del superdotado (pp. 121134). Granada: Aljibe.

Gardner, H. (1995). Mentes creativas. Barcelona, Paidós 1995.

Genovard, C. y Castelló, A. (1990). El límite superior. Aspectos psicopedagógicos de la excepcionalidad intelectual. Madrid: Pirámide

Genovard, C.,Gotzens, C., Badía, M., y Dezcallar, T. (2010). Los profesores de alumnos con altas habilidades. REIFOP, 13(1), 21-31.

Genovard, C.,Gotzens, C., Castelló, A., González, C. y González, P. (1992). Evaluación de procesos cognitivos y optimización instruccional. Infancia y Aprendizaje, 59, 143-152.

Getzels, J. W. (1981). Problem finding and the nature and nurture of giftedness. En A. Kramer (Ed.), Gifted children: Challenging their potential (pp. 1 - 20). New York: Trillium Press.

Gómez, A. Y Valadez, D. (2010). Relaciones de la familia y del hijo/a con superdotación intelectual. Faísca, 15(17), 67-85.

González-Pineda, J., Núñez, J., González-Pumariega, S. y García, M. (1997). Autoconcepto, autoestima y aprendizaje escolar. Psicothema, 9(2), 271-289.

Gross, M. U. (1993). Exceptionally Gifted Children. New York: Routledge. 
Gottfried, A. W., Eskeles, A., Bathurst, K. y Wright, D. (1999). Gifted IQ. Early Developmental Aspects. The Fullerton Longitudinal Study. New York: Plenum Press.

Guilford, J. (1967). The nature of human intelligence. New York: McGraw-Hill.

Guilford, J. (1977). Way beyond the IQ: Guide to improving intelligence and creativity. New York: Creative Education Foundation.

Henson, K. y Heller, B. (2000). Psicología educativa en la enseñanza eficaz. México: Internacional Thompson Editores México

Herrera, F., Ramírez, M., Roa, J. y Herrera I. (2004). ¿Cómo interactúan el autoconcepto y el rendimiento académico, en un contexto educativo pluricultural?. Revista Iberoamericana de Educación. Recuperado de: http://www.rieoei.org/investigacion6.htm

Hoge, R. D. y Renzulli, J. S. (1993). Exploring the link between giftedness and self concept. Connecticut: University of Connecticut.

House, J. (1992). The relationship between academic self-concept and school withdrawal. Journal of Social Psychology, 133(1), 125-127.

Kauffman, J. M. (1995). Why we must celebrate a diversity of restrictive enviroments. Learning Disabilities. Research and Practice, 10(4), 225-238.

Kelly, K. y Colangelo, N. (1984). Academic and social selfconcept of gifted, general and special students. Excepcional Children, 50, 551-554.

Landau, E. (1987). El vivir creativo: Teoría y práctica de la creatividad. Barcelona: Herder.

Landau, E. (1997). Ser superdotado no es sólo ser inteligente, sino que también abarca aspectos sociales y emocionales. Revista Complutense de Educación, 8(2), 33-41.

Landau, E. (2003). El valor de ser superdotado. España: Ministerio de Educación, Cultura y Deporte, Consejería de Educación de la Comunidad de Madrid y Fundación CEIM.

López, M. C. (2003). Análisis de las características y necesidades de las familias con hijos superdotados: propuesta y evaluación de un programa de intervención psicoeducativa en el contexto familiar (Tesis Doctoral). Madrid: Universidad Complutense de Madrid.

Lozano, A. (2003). Factores personales, familiares y académicos que afectan al fracaso escolar en la educación secundaria. Electronic Journal of Research in Educational Psychology, 1(1), 43-66. 
Marsh, H. y Craven, R. (2002). The pivotal role of frames of reference in academic self-concept formation: The "big fish-little pond" effect. En F. Pajares y T. Urdan (Eds.), Academic motivation of adolescents (pp. 83 - 124). Inglaterra: Information Age Publishing.

Martínez, M. y Castelló, A. (2004). Los perfiles de la excepcionalidad intelectual. En S. Castañeda (Ed.), Educación, aprendizaje y cognición. Teoría en la práctica (pp. 251 266). México: Universidad Nacional Autonóma de México / Universidad Autónoma de Guadalajara / Manual Moderno.

Mendez-Sánchez y Palacios-Salas (2001). Estandarización del test de Matrices Progresivas de Raven, escala avanzada (MPA). México: Paidós.

Möller, J. y Pohlmann, B. (2010). Achievement differences and self-concept differences: Stronger associations for above or below average students?. British Journal of Educational Psychology, 80, 435-450.

Monereo, C., Castello, M., Clariana, M., Palma, M. y Pérez, M. (1994). Estrategias de enseñanza y aprendizaje. Formación del profesorado y aplicación en la escuela. Barcelona: Graó.

Mönks, F. (1996). Desarrollo de los adolescentes superdotados. En Y. Benito (Ed.), Desarrollo y educación de los niños superdotados. Salamanca: Amarú.

Mönks, F. (2008). Las necesidades de los hiperdotados: Un modelo óptimo de repuesta. Recuperado de: http://www2.trainingvillage.gr/download/agora/themes/agora09/A9_Monks_ES.pdf.

Mönks, F. y Boxtel, H. (1985). Los adolescentes superdotados: una perspectiva evolutiva. En J. Freeman (Ed.). Los niños Superdotados: Aspectos Psicológicos y Pedagógicos. España: Santillana.

Monreal, C. (2000). Qué es la creatividad. España: Biblioteca Nueva.

Moriana, Alós, Alcalá, Pino, Herruzo y Ruiz (2006). Actividades extraescolares y rendimiento académico en alumnos de educación secundaria. Electronic Journal of Research in Educational Psychology, 4(1), 35-46.

Musitu, G., García, F. y Gutiérrez, M. (2002). Autoconcepto Forma 5 (A. F. 5) Manual. Madrid: TEA Ediciones.

Olszewski, P. y Yasumoto, J. (1995). Factors Afeccting the Academia Choices of Academically Talented Middle School Students. The Journal for the Education of the Gifted 18(3), 299318. 
Ordaz, V. G. y Acle, T.G. (2010). Importancia de la identificación de adolescentes sobresalientes. Ideacción, 31, 321-328. ISSN 1695-7075.

Peñas, F. M. (2008). Características socioemocionales de las personas adolescentes superdotadas. Ajuste psicológico y negación de la superdotación en el concepto de sí mismas. España: Ministerio de Educación, Política, Social y Deporte.

Preckel, F., Goetz, T., Pekrun, R. y Klein, M. (2008). Gender diferences in gifted and average ability students: Comparing girls' and boys' achievement, self-concept, interest, and motivation in mathematics. The Gifted Child Quarterly, 52(2), 146-159.

Puga, I. (2004). Los niños diferentes, con más capacidades y habilidades. Educar, 43, Abril Junio.

Raven, J. C. (1993). Test de Matrices Progresivas: Cuaderno de Matrices, Serie I. Madrid: Paidós.

Raven, J. C., Court, J. H. y Raven, J. (2008). Manual de Test de Matrices Progresivas. Escalas coloreada, general y avanzada. Bueno Aires: Paidós.

Rayo, L. J. (1997). Necesidades Educativas del Superdotado. España: EOS.

Reis, S. M. (2003). Gifted Girls. Twenty-five years later: hopes realized and new challenges found. Roeper Review, 25(4), 154-157.

Reis, S. M. y Renzulli, J. (2004). Current research on the social and emotional development of gifted and talented student: good news and future possibilities. Psychology in the Schools, 41(1), 119-130.

Renzulli, J. (1978). What makes giftedness?: Reexamining a definition. Phi Delta Kappan, 60, $180-184$

Renzulli, J. (1994). El concepto de los tres anillos de la superdotación. Un modelo de desarrollo para la productividad creativa. En M. Y. Benito (Ed.), Intervención e investigación psicoeducativas en alumnos superdotados (pp. 171 - 217). España: Amarú.

Renzulli, J. (2002). Emerging conceptions of giftedness: building a bridge to the new century. Exceptionality, 10(2), 67-75.

Renzulli, J. y Park, S. (2000). Gifted dropouts: The who and the why. Gifted Child Quarterly, 44(4), 261-271.

Richert, E. S. (1997). Excellence with equity in identification and programming. En N. Colangelo y G. Davis (Eds.), Handbook of gifted education (pp. 75 - 88). USA: Allyn and Bacon. 
Robinson, N. y Olzewski-Kubilius, P. (1997). Niños superdotados y talentosos: temas para pediatras. Pediatrics in Review, 18.

Rodríguez, R. I. (2002). Factores extrínsecos que inciden en el rendimiento deficitario de las mujeres. En L. Pérez; L. Domínguez y E. Alfaro (Eds.), Actas del seminario: situación actual de la mujer superdotada en la sociedad (pp. 87 -102). Madrid: Comunidad de Madrid Consejería de Educación.

Rojo, A. (1999). La Teoría Triárquica de la Inteligencia: Un Nuevo Enfoque para el Estudio y la Valoración del Superdotado. En D. Prieto (Ed.), Identificación, Evaluación y Atención a la Diversidad del Superdotado (pp. 59 - 77). Málaga, España: ALJIBE.

Santana, L., Feliciano, L. y Jiménez, A. (2009). La influencia del autoconcepto en el rendimiento académico en estudiantes universitarios. REOP, 20(1), 16-28.

Schunk, D. (1991). Self-efficacy and academic motivation. Educational Psychologist, 26, 207231.

Secretaría de Educación de la Ciudad de México (2008). Entrega de computadoras y niñas y niños talento. México: Boletín 059-8.

Secretaría de Educación Pública (2006). Propuesta de intervención: Atención educativa a alumnos y alumnas con aptitudes sobresalientes. México: SEP.

Secretaría de Educación Pública (2008). Sistema Educativo de los Estados Unidos Mexicanos: Principales cifras del ciclo escolar 2007-2008. México: SEP.

Silverman, L. K. y Kearney, K. (1988). Parents of the extraordinarily gifted. Advanced Development, 1, 1-10.

Simonton, D. K. (1994). Greatness: Who makes history and why?. New York: Guilford.

Snowden, P. L. y Christian, L. G. (1997). Parenting the young gifted child: supportive behaviors. Roeper Review, 21(3), 215-221.

Sternberg, R. J. (1996). Successful intelligence. New York: Simon \& Schuster.

Tannembaum, A. J. (1983). Gifted Children: Psychological and educational Perspectives. New York: Macmillan.

Tannembaum, A. J. (1997). The meaning and making of giftedness. En N. Colangelo y G.A. Davis (Eds.), Handbook of gifted Education. Boston: Allyn \& Bacon.

Terman, L. M. (1925). Genetic studies of genius: Mental and physical traits of a thousand gifted children. Stanford, CA: Stanford University Press. 
Torrance, E. P. (1962). Guiding creative talent. Englewood Cliffs, NJ: Prentice-Hall.

Torrance, E. P. (1984). The Role of Creativity in Identification of the Gifted and Talented. Gifted Child Quarterly, 28(4), 153-156.

Torrance, E. P. (2008a). Thinking Creatively with Pictures: Figural Response Booklet A. Bensenville, Ill: Scholastic Testing Service, Inc.

Torrance, E. P. (2008b). Torrance Tests of Creative Thinking: Norms-Technical Manual Figural (Streamlined) Forms A\& B. Bensenville, Ill: Scholastic Testing Service.

Torrance, E. P., Ball, O. E. y Safter, H. T. (2008). Torrance Tests of Creative Thinking: Streamlined Scoring Guide for Figural Forms A and B. Bensenville, Ill: Scholastic Testing Service.

Touron, J., Peralta, F. y Repáraz, C. (1998). La superdotación intelectual: Modelos, identificación y estrategias educativas. España: Ediciones Universitarias de Navarra, S. A.

Valadez, D. (2004). Niñas, superdotación y contexto social. Educar, 43, Abril - Junio.

Vidals, A. (2005). Autoconcepto, locus de control y rendimiento académico en estudiantes de segundo semestre de la facultad de psicología (Tesis de maestría). Universidad Nacional Autónoma de México, México.

Zacatelco, F. (2005). Modelo para la identificación del niño sobresaliente en escuelas de educación primaria (Tesis Doctoral). México: UNAM.

Zacatelco, F. y Acle, G. (2009). Modelo de identificación de la capacidad sobresaliente. Revista Mexicana de Investigación en Psicología, 1(1), 41-43.

Zavala, M. A. (2004). La detección de alumnos superdotados en las escuelas primarias (Tesis Doctoral). México: Universidad Autónoma de Aguascalientes.

Zavala, M. A. y Rodríguez, J. L. (2004). El campo educativo de niños y jóvenes con aptitudes sobresalientes. Relieves históricos y problemática actual. Educar, 43, Abril - Junio. 